\title{
Gene Editing Versus Morphants
}

\author{
Paul A. Morcos, ${ }^{1}$ Alexandra C. Vincent, ${ }^{2}$ and Jon D. Moulton ${ }^{2}$
}

\section{Dear Editor:}

$\mathbf{K}$ OK ET $A L$. conclude that results with Morpholino oligos, which are not recapitulated by natural mutations or gene editing mutations, must be due to off-target effects and, when morphants and mutants differ, they contend that antisense oligo studies should be disregarded. ${ }^{1}$ Morpholinos and gene editing mutagenesis are very different technologies and, even if both work perfectly, may yield different results targeting the same gene. Rossi et al. showed in zebrafish with a gene either knocked out or knocked down that some untargeted genes altered their expression in the mutant but not the morphant. ${ }^{2}$ The morphants showed a strong phenotype but the mutants, with their compensatory expression changes, did not show the phenotype. Another knockdown technique, CRISPRi, phenocopied the Morpholino result. They further showed that when Morpholinos were used in zebrafish heterozygous for the mutation, the morphant phenotype was partially suppressed, which was not expected if the morphant phenotype were primarily due to off-target RNA interaction. Gene editing technologies cause permanent changes in DNA. Morpholinos work on RNA, can attenuate targets that may be lethal when knocked out with gene editing, and cause transient and concentration-dependent knockdowns. We argue that different reported outcomes of Morpholinos and gene editing may be due to the differences in timing and site of action in addition to overdosing, lack of p53 controls, and lack of specificity controls when using Morpholinos.

Antisense oligos can give rise to off-target effects, especially at high concentration. However, screening Morpholino target sequence with BLAST and using proper dosing routinely yields valuable and reproducible results. To showcase their hypothesis, Kok et al. describe a phenotype from a Morpholino used in a megamind null mutant. ${ }^{1}$ The MO dose was $20 \mathrm{ng} / \mathrm{embryo}$, higher than the 5ng dose Ulitsky et al. reported for their megamind morphant. ${ }^{3}$ Further, Lin et al. used both splice-targeted and conservedregion-targeted Morpholinos against megamind at $1 \mathrm{ng}$ and found no morphological phenotype. ${ }^{4}$ Notably, $1 \mathrm{ng}$ caused a behavioral phenotype and made the wild-spliced RT-PCR gel band disappear; while in the megamind mutant this phenotype has not been reported, behavior might not have been assessed. Lin et al. show a megamindtargeted Morpholino alters RNA processing and yields phenotypic data when used at a dose below that causing hydrocephaly; Kok et al. did not report testing a series of lower doses.

Kok et al. continue presenting the merits of gene editing technologies by comparing selected experimental results for specific genes: (a) whose RNA transcripts are blocked by Morpholinos, versus (b) whose DNA sequences are partially or fully deleted by gene editing technologies. ${ }^{1}$ They conclude that the vast majority of published Morpholino results are due to off-target effects because the Morpholino and gene editing results do not match, however Rossi et al. show compensation might cause the difference. ${ }^{2}$ In addition, most of the Morpholino work chosen for comparison did not report controlling for specificity by phenocopying using nonoverlapping Morpholinos targeted to the same RNA and did not report screening for activation of the p53-mediated apoptosis cascade. Oligo specificity is commonly confirmed by phenocopying with non-overlapping Morpholinos or using rescue mRNAs. Morpholinos confirmed with these techniques and used at optimized dosage are very specific for their targeted RNA sequences.

Kok et al. advocate rejecting Morpholino results unless a knockdown phenotype is confirmed against a mutant. ${ }^{1}$ This is causing some reviewers of papers and grants to discount Morpholino experiments and insist DNA manipulations be used instead of or in addition to Morpholinos. We believe this is counter-productive for the developmental biology community. Generating morphants with Morpholinos is a well-validated technology with solid targeting rules and multiple strategies available for rigorously confirming results.

\section{References}

1. Kok FO, Shin M, Ni C-W, Gupta A, Grosse AS, van Impel A et al. Reverse genetic screening reveals poor correlation between morpholinoinduced and mutant phenotypes in zebrafish. Dev Cell. 2015;32(1): 97-108.

2. Rossi A, Kontarakis Z, Gerri C, Nolte H, Hölper S, Krüger M, Stainier DYR. Genetic compensation induced by deleterious mutations but not gene knockdowns. Nature. 2015; [Epub ahead of print] doi:10.1038/nature 14580 .

3. Ulitsky I, Shkumatava A, Jan CH, Sive H, Bartel DP. Conserved function of lincRNAs in vertebrate embryonic development despite rapid sequence evolution. Cell. 2011;147(7):1537-50.

4. Lin N, Chang KY, Li Z, Gates K, Rana ZA, Dang J et al. An evolutionarily conserved long noncoding RNA TUNA controls pluripotency and neural lineage commitment. Mol Cell. 2014;53(6):1005-19.

Address correspondence to: Paul A. Morcos, PhD OligoExpert 553 NW Mountain Laurel Circle Corvallis, OR 97330

E-mail: pmorcos@oligoexpert.com

\footnotetext{
${ }^{1}$ OligoExpert, Corvallis, Oregon.

${ }^{2}$ Gene Tools, LLC, Philomath, Oregon.
}

(C) The Paul A. Morcos et al. 2015; Published by Mary Ann Liebert, Inc. This Open Access article is distributed under the terms of the Creative Commons License (<http://creativecommons.org/licenses/by/4.0 $>$ ), which permits unrestricted use, distribution, and reproduction in any medium, provided the original work is properly credited. 